\title{
"WHEN THE NIGHT WIND SOFTLY BLOWS THROUGH MY OPEN WINDOW...« EXZESS UND EXORZISMUS DER GESTE IN HANS-ChRISTIAN SCHMIDS FILM REQUIEM
}

\author{
STEPHAN TRINKAUS
}

Wir hören schnellen Atem, das Keuchen eines Körpers, dann sehen wir eine Rad fahrende junge Frau, sie erreicht eine kleine Kapelle auf einem Berg und klammert sich an das den Altarraum umgebende Gitter: »Bitte «, stößt sie hervor, mehr erfahren wir nicht. So beginnt REQUIEM, ein Film von Hans-Christian Schmid, der 2006 im Wettbewerb der Berlinale gezeigt wurde und kurze Zeit später in die deutschen Kinos kam. Der Film erzählt seine Geschichte - wie es heißt - frei nach einer wahren Begebenheit. Die Pädagogikstudentin Anneliese Michel aus Klingenberg wurde von Krämpfen heimgesucht, hörte Stimmen und wurde schließlich von der katholischen Kirche offiziell als vom Teufel Besessene bezeichnet und mit Genehmigung des zuständigen Bischofs von zwei Pfarrern exorziert. 1976 starb sie nach monatelangen Exorzismen an Unterernährung. Der Film spielt stattdessen in Tübingen und der schwäbischen Alb und aus Anneliese Michel aus Klingenberg wird Michaela Klingler, die Zeit der Handlung allerdings bleibt: in außerordentlicher Genauigkeit stellt der Film die 1970er Jahre nach, wobei er trotz seines Detailreichtums nie zum Kostümfilm wird. Vielmehr entwickelt er so etwas wie einen quasi-dokumentarischen Stil, der mitunter näher und genauer an, ja in der Zeit ist, als es ein Film aus der Zeit selbst je sein könnte. Er spielt - so könnte man vielleicht sagen - in der Immanenz dieser Zeit: es gibt in diesem Film keinen Hinweis, wie das, was wir sehen, jemals über sich hinaus gelangen könnte, keinen Hinweis auf eine mögliche Zukunft. Und dennoch tritt er in eine Konstellation mit unserer Zeit: es ist, als ob erst jetzt, im Jahr 2006, diese Immanenz der siebziger Jahre sichtbar wird, als ob wir erst jetzt, in diesem Augenblick, in eine Zeitgenossenschaft mit ihr eintreten, weit weg von den Bildern der langen Haare und gebatikten Hemden, in der der Moment der Unbestimmtheit, der Kontingenz dieser wie unserer Zeit aufblitzt, der in den zu Emblemen geronnenen Bildern 
der Revolte geradezu ausgeschlossen bleibt. Darin antwortet REQUIEM meines Erachtens auch Hans-Christian Schmids letztem Film LicHTER, der dieser Unbestimmtheit in einigen Episoden über die Illusionen und Hoffnungen der Bewohner oder eher Reisenden des Grenz- und Übergangsraums zwischen Stubice und Frankfurt auf der Spur war.

Doch zurück zu REQUIEM: In der Szene, die auf die oben erwähnte Eingangssequenz folgt, erfahren wir bereits, worum die junge Frau in der Kapelle gebeten hat und dass der Gott, den wir mit ihr jenseits des Gitters vermuten, ihr diese Bitte zu gewähren scheint: Die Zusage für einen Studienplatz in Tübingen, mit dem sie die Türen ihrer engen Heimat, einem kleinen Dorf in der schwäbischen Alb, weit aufstoßen kann. Doch etwas stimmt nicht mit diesem Gott hinter dem Gitter, der diesen Horizont öffnet und einen Weg, einen eigenen Weg, zu ermöglichen scheint. Es ist, als ob er die Tür, die er öffnet, in der gleichen Bewegung wieder schließt: Michaelas Weg nach Tübingen und ins Studium wird skandiert von einem jähen Wiederaufleben ihrer Krankheit, die die Ärzte als Epilepsie diagnostiziert haben und die durch Tabletten beherrschbar schien. Hinzu kommen Stimmen, die sie heimsuchen und davon abhalten den Rosenkranz oder das Kruzifix zu berühren, ihr das Beten unmöglich machen. Alle Praktiken also, die zuvor der Herstellung der eigenen Stabilität dienten, der religiösen Verortung und Regulierung des Körpers scheinen von nun an in eine radikale Instabilität zu führen: Die Öffnung, das Leben in Tübingen, das Erleben des eigenen Begehrens können weder von den Symbolen und Ritualen der Herkunft noch von den chemischen Substanzen der Klinik gebunden werden, sie machen den Körper Michaelas vielmehr zum Schauplatz einer Konfrontation, der gewaltige Mengen an Energie zur Verfügung zu stehen scheinen. In dieser eskalierenden Gleichzeitigkeit von Öffnung und Schließung, Freiheit und Zwang, Begehren und Verbot sitzt Michaela, man müsste wohl eher sagen, der Körper Michaelas fest, immer wieder aufbrechend, immer wieder zurückgeworfen.

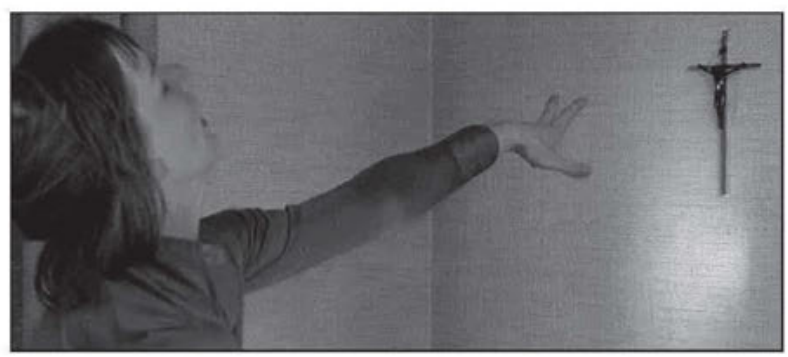

Abb. 1: REQUIEM (2007) 
Im Film erscheint das im zitternden \Stillstand jener gewaltsamen Gesten, in denen Michaela nach dem Kruzifix oder dem Rosenkranz, den die Mutter ihr geschenkt hat, zu greifen versucht: Auf halbem Wege bleibt sie stecken in einem krampfhaften, gewaltsamen Zeigen. Es ist hier nicht mehr klar, welche Bewegung dieses chiastischen Steckenbleibens eigentlich worauf hinaus will. Ist es die Intentionalität des >Greifenwollens〈, die Freiheit, Selbstbestimmung, Autonomie bedeutet, die gewaltsam davon abgehalten wird, sich zu realisieren? Oder liegt der Zwang, die Gewalt nicht gerade in dieser Intention, die aus der Hand der Mutter kommenden Gegenstände zu berühren, sich ihnen zu unterwerfen? Diese Ununterscheidbarkeit von Freiheit und Zwang, Macht und Ohnmacht führt jedenfalls in einen Zustand, der sich in keine Richtung mehr auflösen lässt. Die Art und Weise, in der sie sich auf diese Welt bezieht, in der sie in diese Welt verknüpft ist, produziert eine Bewegung, die sich nur noch auf sich selbst bezieht, ein Wollen, das zugleich Nichtwollen, ein Können, das zugleich Nichtkönnen ist. Diese angespannte, bebende Bewegung in der Nichtbewegung mündet - so legt es der Film nahe - in einen Zustand vollkommenen »Außer-sich-Seins«, dem 〉Grand Mal« des epileptischen Anfalls. Doch davon zeigen uns die Bilder nichts: Der Moment des Anfalls ist der Moment des Schnitts, jener Moment also, der zugleich Bruch und eigentliche Bewegung des Kinos ist.

Die Verbindung von Epilepsie und Besessenheit hat eine lange Geschichte in der christlichen Kultur des Abendlands. Ich habe nicht das Wissen, um sie hier im Einzelnen nachzeichnen zu können. Vor allem möchte ich aber vermeiden, bei diesem Thema in eine Falle zu gehen, der sich auch der Film konsequent verweigert: sich in der Rekonstruktion einzelner religiöser Anspielungen und Symbole selbst in jenen verführerischen Mystizismus einspinnen zu lassen, der schließlich im Exorzismus endet. Dasselbe gilt natürlich auf etwas andere Weise auch für den klinischen Diskurs. Dennoch möchte ich auf eine Episode ganz zu Beginn dieser gemeinsamen Geschichte hinweisen, die ich für äußerst aufschlussreich halte: die direkt auf die Szene der »Verklärung Jesu« auf dem Berg Tabor folgende Episode von der Heilung des besessenen Knaben, wie sie im Evangelium nach Markus (Markus 9: 2-29) erzählt wird. Die Transfigurationsepisode beginnt folgendermaßen:

»Jesus [nahm] Petrus, Jakobus und Johannes beiseite und führte sie auf einen hohen Berg, aber nur sie allein. Und er wurde vor ihren Augen verwandelt; seine Kleider wurden strahlend weiß, so weiß, wie sie auf Erden kein Bleicher machen kann.« Danach erscheinen Elija und Moses, die Jünger bauen drei Hütten, eine für Elija, den jüdischen Propheten, eine für Moses und eine für Jesus. Dann spricht Gott aus einer Wolke: »Dieser ist mein geliebter Sohn, auf ihn sollt ihr hören.« Während des 
Abstiegs kommt es zu einem Gespräch über die Prüfungen, die ihm, Jesu, bevorstünden und er gebietet seinen Jüngern von dem Gesehenen zu schweigen, »bis der Menschensohn von den Toten auferstanden sei«. Zurück am Fuß des Berges bei den restlichen Jüngern tritt ein Vater mit seinem besessenen Sohn vor ihn: »Immer wenn der Geist ihn überfällt, wirft er ihn zu Boden und meinem Sohn tritt Schaum vor den Mund, er knirscht mit den Zähnen und wird starr«. Der Vater fleht: »Wenn du kannst, hilf uns; hab Mitleid mit uns! « und Jesus antwortet: »Wenn du kannst? Alles kann wer glaubt.« Also zwingt Jesus den Geist dazu, aus dem Körper des Besessenen auszufahren.

»Da zerrte der Geist den Jungen hin und her und verließ ihn mit lautem Geschrei. Der Junge lag da wie tot, so dass alle Leute sagten: Er ist gestorben. Jesus aber fasste ihn an der Hand und richtete ihn auf, und der Junge erhob sich. Als Jesus nach Hause kam und sie allein waren, fragte ihn einer seiner Jünger: Warum konnten denn wir den Dämon nicht austreiben? Er antwortete ihnen: Diese Art kann nur durch Gebet ausgetrieben werden.«

Die Episode von der Heilung des Besessenen scheint die Geschichte Jesu, die Leiden und die Auferstehung von den Toten vorwegzunehmen, sein Zustand - so wird es hier von Markus wohl nahe gelegt - unterhält eine geheime Beziehung zur Verklärung Jesu, so als ob die Besessenheit das negative Pendant zur Transfiguration sei, in der die durch das Opfer des Menschensohns erreichte Erlösung vorweg genommen wird.

Raffael hat diese beiden Szenen zu Beginn des 16. Jahrhunderts in einem berühmten Gemälde - DIE VERKLÄRUNG ODER TRANSFIGURATION CHRISTI - zusammengefasst. Auf diesem Gemälde, Raffaels letztem, erscheint innerhalb einer äußerst komplexen Figurenkonstellation ein verklärter, blendend heller Christus mit deutlich weiblichen Formen, der über einem Berg in Wolken schwebt. Er wird hier konstelliert mit dem Körper eines in Konvulsionen erstarrten Jungen mit nach innen gekehrten Augen und einer Frau im Vordergrund, deren entblößte Schulter und deren Hintern mindestens so hell zu leuchten scheinen, wie der verklärte Christus. Während der leuchtende Leib Jesu mit seinem ausladenden Becken die obere Bildhälfte beherrscht, steht die Frau im Zentrum der unteren Hälfte. Es ist aber der Körper des Jungen, auf den gezeigt und der betrachtet wird - so als ob er die Schaltstelle sei, in der die untere auf die obere Welt verwiesen ist.

Es wäre sicherlich äußerst interessant, dieser komplexen Konstellation im Einzelnen nach zu gehen, den unterschiedlichen Bewegungen der Hin- und Abwendung, der verschiedenen Gesten. Was das Bild jedoch vor allem auszeichnet, ist das üppige und prächtige Spiel der Farben und Falten, in deren Zentrum die Gewänder stehen, die den Frauenkörper 
umspielen und hervorheben. In den Farben und Faltungen der Gewänder erscheint etwas, das mit dem überirdischen Leuchten der Jesus-Gestalt in Verbindung zu stehen scheint: die Präsenz eines weiblich konnotierten Begehrens, das über die einzelnen Figuren, ihre Figürlichkeit hinausgeht. Der Körper des Jungen, obwohl augenfällig eine zentrale Gestalt, fällt jedoch aus dieser Präsenz heraus: Er ist fast nackt, seine angespannte Bewegung alles andere als anmutig. Während die Frau, die Farben und die Falten so etwas wie der Widerschein der Weiße Jesu zu sein scheinen, so ist der Junge vielleicht sein Antipode, seine Rückseite: der verkrampfte männliche Körper in dem das leichte, wallende Leuchten in eine angestrengte Anspannung umschlägt, sich auf eine Weise konzentriert, die den Jungen von innen her in ein Außen des Bildes zu ziehen scheint. So wie Jesus zu schweben beginnt, so scheint der Junge mit seinen krampfhaft ausgestreckten Armen eingespannt zwischen dem Oben und dem Unten, dem Diesseits und dem Jenseits. Geht man den Blicken und Gesten in der unteren Bildhälfte nach, so stellen sie (beinahe) alle eine Verbindung zwischen der Transfiguration und dem Anfall des Jungen her. Und es ist fast als spiegele sich die Weiße Jesu in der Weiße der nach innen gedrehten Augäpfel des Jungen: Ein Blick, der aus der Welt hinaus und in den Körper hinein geht, in das absolute Außen eines absoluten Innen. Innerhalb dieses Bildes, und das scheint mir hier zentral, wird es keinen Exorzismus, keine Heilung geben. Die Konstellation, die es ausstellt, läuft auf keine Erlösung hinaus. Jesus wird hier nicht herabsteigen und mit der Macht des Glaubens und des Gebets die Dämonen ausfahren lassen, seine Transfiguration findet hier gleichzeitig mit der Besessenheit statt, steht in einer Spannung, ja einer Abhängigkeit zu ihr. Ohne dieses Außen - so scheint es - würde das Leuchten der Transfiguration aus der Welt verschwinden.

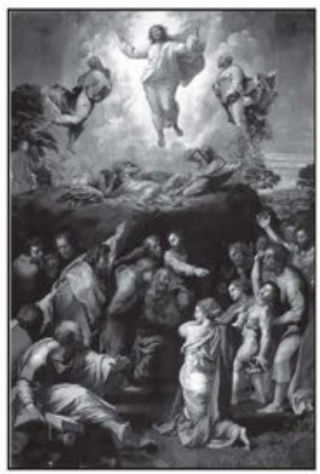

Abb. 2: TRANSFIGURATION (1517) 
Das ganze Bild ist meines Erachtens so etwas wie eine Präsentation des Gestischen, des Gestischen als dem Moment des, wie Giorgio Agamben es ausdrückt, »In-der-Sprache-sein des Nichtsprachlichen« (Agamben 2003: 71), oder anders: des Chiasmus der unauflöslichen Verflochtenheit des Körpers in das, was Merleau-Ponty das »Fleisch der Welt« genannt hat und seiner gleichzeitigen Unbindbarkeit. Während sich in den Farben und Faltungen, den ausladenden Becken dieses >Fleisch (figuriert bis hin zu seiner Transfiguration in der Weiße Jesu, so ist der Körper des besessenen Jungen, der hier ausgestellt, der vorgezeigt wird, das Außen des Körpers, des Nichtsprachlichen. Das Bild präsentiert eben den Moment, in dem sich beides begegnet, einen Moment der Kontingenz und der Potentialität, in dem die Ordnung der Welt ihre eigene Materialität berührt. Das Reich der Geste ist diese Berührung zwischen Bindung und Entbindung, Sprachlichem und Nichtsprachlichem, Medialität und Materie. Es erstreckt sich von der reinen Bindung und Übergängigkeit der Transfiguration bis zum radikalen Außen der Besessenheit. All die Faltungen und Farben, die Hin- und Abwendungen, das Abschirmen und Niederwerfen, Zeigen und Verbergen finden in dieser Gleichzeitigkeit, dieser Spannung, dieser Berührung statt. Das heißt in der frühneuzeitlichen Konstellation des Raffaelschen Bildes: Die Geste bezeichnet hier zugleich den Moment der Unerlösbarkeit als auch die Möglichkeit der Erlösung, ja sie ist dieser Moment, in dem die Welt sich ihrer eigenen Kontingenz ausliefert und so die Erwartung, das Offene, die Messianizität in der Welt hält.

Wie sieht es jedoch mit den Gesten des konvulsiven Körpers von Michaela in REQUIEM aus? Im Film steht dieser Körper im Zentrum wohlmeinender Bemühungen, ihn zu normalisieren, ihn zurückzuholen auf seinen Weg vom kleinen Dorf in die Provinzstadt, vom Kinderglauben in die Verantwortung für das eigene Leben. Die Freundin ruft nach der Klinik: "Jetzt akzeptier doch endlich, dass du krank bist«, doch die hat die junge Frau schon hinter sich, ein Jahr hat sie verloren, nichts hat es ihr gebracht. Der Vater verlangt: $» S a g,[\ldots]$ dass es dir gut geht und dass du gelernt hast. Dass es viel Arbeit ist und dass du deine Tabletten nimmst und dass hier Scheissdreck geredet wird. Dass du müde bist und mal ausschlafen musst und nicht weißt, was du sprichst. Sags.« (Lange 2006: 141) Auch der Dorfpfarrer: »Hörst du dich reden? Fratzen und Stimmen. Von was sprichst du? Vom Teufel? [...] Aber das sind Sinnbilder, Beispiele [...] das kannst du doch nicht Wort für Wort nehmen« (Lange 2006: 60), der engagierte Jungpfarrer, der einzige, der etwas zu verstehen scheint, der Michaela zumindest für einen Moment einzufangen scheint, ihr das Martyrium, die Opferrolle, das sinngesättigte Leiden, das geradezu klassische auf die Erlösung gerichtete Begehren des katholischen 
weiblichen Körpers bietet und schließlich der Freund, der sie begleitet, wenig fordert, ihr ein Schäfchen - ein Lamm, um genau zu sein - zu Weihnachten schickt, dem aber bald »alles zu viel« ist. Alle versuchen der Erfahrung Michaelas, dem, was sich in ihrem Körper, durch ihren Körper ereignet aus dem Weg zu gehen. Man beginnt unwillkürlich sich zu fragen, welche Strategien man selbst gefunden hätte, um dieser Erfahrung auszuweichen.

Aber dann gibt es noch die Mutter: Die Dämonen, die sie von der mütterlichen Gabe, dem Rosenkranz, abzuhalten versuchen, scheinen mit ihrer Stimme, der Stimme der Mutter, der Muttersprache, zu sprechen. Man sieht sie nicht und man hört sie eigentlich auch nicht, aber wenn die junge Frau zu schreien beginnt, so meint man die Stimme der Mutter herauszuhören: »Dreckschleuder «, »Saustall«. Es ist wohl das Begehren der Mutter, das Michaela einsperrt, über das sie nicht hinauskommt, ein Begehren, das jede ihrer Gesten heimsucht und es am Ende unmöglich macht, die mütterliche Anwesenheit sei es in Gestalt des Rosenkranzes oder auch des Kruzifixes zu ertragen und zu den unerträglichen sich entladenden Stasen des Anfalls führt. Diese Mutter ist eine Mutter des Verwurzeltseins, der radikalen Verortung, der Bewegungslosigkeit: Ihre Gesichtszüge sind starr, aus ihr blickt nichts zurück, sie wirft die neuen Kleider ihrer Tochter in den Müll, damit sie nicht aussieht wie ein »Flüchtlingskind«. Die Enge der Welt, in der Michaela aufwächst, erscheint zuallererst als ihre Enge. Sie ist das energetische Zentrum der Familie, diejenige, die deren Struktur aufrecht erhält, die es auf sich genommen hat, die Kontingenz zu verschlucken in einer Krypta, in der sie auch ihre Töchter festzuhalten versucht. Ihr tiefer Hass auf jede Form von Bewegung ist in jeder Szene spürbar, ihre Abwehr und ihre Angst.

Julia Kristeva hat in »Fremde sind wir uns selbst« darauf hingewiesen, dass sich der Heimatlose und der Verwurzelte einander gegenüber stehen: »Eine scheinbar friedliche Koexistenz, die den Abgrund verbirgt: eine kaputte Welt, das Ende der Welt.« (Kristeva 1990: 27) Dieser Abgrund der verschluckten und in der Mutter kryptisierten Kontingenz geht durch den Körper der Tochter hindurch: In der Verwurzelung wohnt eine Fremdheit, die so fremd ist, dass sich daraus keine Bedeutungen, kein Weg mehr hervorbringen lassen. In dem Moment also, in dem sich diese Welt öffnet, erscheint der Abgrund, die Kontingenz, die Instabilität des Körpers, die Geste.

»Die Beziehung zur Welt ist eine Beziehung der Präsenz in der Welt, des in der Welt Seins im Sinne des der Welt Angehörens, ja des von ihr Besessenseins, eine Beziehung, in der weder Akteur noch Gegenstand als solche definiert sind " (Bourdieu 2001: 180), schreibt Pierre Bourdieu in seinen Pascalschen Meditationen. Die Geste ist sicherlich diese Be- 
ziehung der Präsenz in der Welt, ein Ausgeliefertsein an die Dinge und den Anderen über alle Aktivität und Passivität hinaus, eben »Besessenheit«. Doch was, wenn diese Welt, von der wir besessen sind, zum Abgrund wird, zum Ende der Welt, wie Kristeva sagt, die Präsenz in der Welt zur Präsenz des Endes der Welt? Hier tut sich eine in der maßlosen Fixierung der Verortung unbindbare Ortlosigkeit auf: Wenn die Geste in dieser Konstellation erscheint, erscheint sie als ihr eigener, sich selbst überbordender und immer weiter treibender Exzess, als unbindbare göttliche Gewalt, die über und durch den Körper kommt. Darin besteht wohl der Zusammenhang zwischen dem bebenden Stillstand und dem Anfall: Sie bezeichnen das Unmäßigwerden der Geste, einen Exzess des Gestischen, der auf die eigene Auslöschung aus ist. Der Körper fällt von Kataklysmus zu Kataklysmus: unregulierbar, unbindbar.

»Was in jedem Ausdruck ohne Ausdruck bleibt, ist Geste«, schreibt Giorgio Agamben zu diesem Paradox im Anschluss an Benjamins Hölderlin-Lektüren (Agamben 2004: 46). Ganz kann ich mir den etwas überdeutlichen Hinweis nicht verkneifen: der Film spielt nicht nur in der Ortlosigkeit des Orts, sondern auch in Tübingen...

Es gibt zwei Szenen in REQUIEM, an denen dieser Körper zu sich selbst zu kommen scheint, an denen er eine große Ruhe ausstrahlt, einen Glanz, ein Leuchten: Immer wenn diese schreckliche Siebziger-JahreOrgel zu wimmern beginnt (die in Wahrheit aus den 1960ern stammt), aus der die ganze Weite und die ganze Enge dieses Jahrzehnts und dieses Ortes anklingt und eine tiefe, wohltönende Männerstimme anhebt: »When the night wind softly blows through my open window...«. Beim ersten Mal geschieht das nach dem wahrscheinlich ersten Kuss, der ersten Umarmung. Der Körper beginnt zu tanzen und es ist, als ob er auch das erste Mal tanzen würde. Dieser Tanz - die kleine Schwester der Epilepsie, wie jemand im Internetforum von REQUIEM angemerkt hat - ist sicherlich der eigentliche Höhepunkt des Films: der Partykeller, die Lichtorgel, Deep Purple und dieser tanzende Körper, der mit jeder Bewegung freier, strahlender, schwebender wird, der sich aus dem beengenden Raum herauszutanzen scheint, die Bilder der Zeit und des Orts hinter sich lassend, schließlich sich selbst hinter sich lassend. Dann Schnitt - man sieht noch die Rückkehr in das Studentenwohnheim Schnitt - das erschöpfte und von einem glücklichen Seufzen begleitete ins Bett Fallen und - Schnitt - es ist bereits hell, die Freundin kommt über den Flur, ist verärgert, weil sie nicht geweckt wurde, tritt in das Zimmer mit dem Kruzifix über dem Schreibtisch und darunter liegt sie, zusammengekauert, krümmt sich, will sich nicht anfassen lassen, schreit.

Ganz am Ende des Films kehren diese Orgel, diese Stimme und diese Zeile wieder und wir sehen die junge Frau auf einer Bank, auf einem 
Hügel neben ihrer Freundin sitzen. Es scheint der gleiche Ort wie zu Beginn, doch von einer Kapelle ist nichts mehr zu sehen. Beide blicken in die Weite der schwäbischen Alb, deren Felder - so die Freundin - aussehen »wie eine Patchworkdecke«. Die Freundin möchte Michaela von hier wegbringen, heraus aus dem Dorf, aus dem auch sie kommt und wo sie geschlagen wird von ihrem alkoholkranken Vater, einem Außenseiter der Dorfgemeinschaft, auf den Michaelas Eltern nicht gern zu sprechen kommen. Doch wohin? Sicher, nach Tübingen, aber was bedeutet das? Die Freiheit? Die Klinik? Michaela möchte nicht weg, sie scheint bereit, dem, was sie für ihre Bestimmung hält, entgegenzugehen oder eher - sie möchte aufhören überhaupt irgendwo hin zu gehen, das scheint nun »ihr Weg«. Ein Weg, der die Bewegungslosigkeit, das Nirgendwo-Ankommen aufnimmt und in das Außen des Innen führt: "Man kann es sich nicht aussuchen, was Gott mit einem vorhat. Ich muss meinen Weg gehen. Bringst du mich wieder zurück? « (Lange 2006: 180) Es folgt eine Fahrt im Auto. Wir sehen von außen durch das Autofenster das Gesicht der jungen Frau und das vorbeigleitende Geäst der Bäume, den Himmel sich im Glas der Scheibe und dem Gesicht dahinter spiegeln, Formen und Figuren bilden, die sich wieder auflösen, neue Gestalten annehmen ohne sich je zu fixieren. Michaela tritt mit uns ein ins Reich der Geste und eine unglaubliche Ruhe geht von ihrem sich ständig wandelnden Gesicht hinter der Scheibe aus, eine provozierende Harmonie - die scheußliche Orgel hebt an und die Stimme beginnt von neuem: »When the night wind softly blows through my open window... Und so wie das Keuchen dem Film vorausgeht, seine Bilder hervorzubringen scheint, so überlebt dieser Gesang, diese Orgel, diese Zeile das Bild des Körpers, als ginge er über in die muffige Weite dieser Musik, die immer schöner, immer größer zu werden beginnt: Das Bild wird schwarz und der Abspann erscheint.

Die Geschichte, die der Film erzählt, endet empörend: »Nach einer Serie von mehreren Dutzend Exorzismen«, so der Abspann, »verstirbt Michaela Klingler an Entkräftung im Haus ihrer Eltern« (Lange 2006: 182).

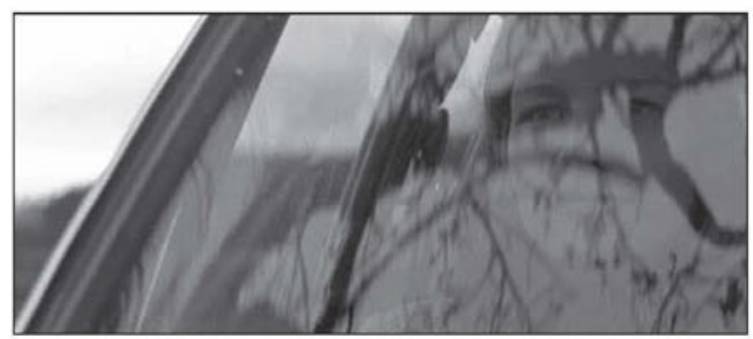

Abb. 3: REQUIEM (2007) 


\section{Literatur}

Agamben, Giorgio (2003): Die kommende Gemeinschaft, übers. v. Andreas Hiepko, Berlin: Merve.

Agamben, Giorgio (2004): »Noten zur Geste«. In: Hemma Schutz/Tanja Widmann (Hg.): Dass die Körper sprechen, auch das wissen wir seit langem, Köln: Walther König, S. 39-48.

Bourdieu, Pierre (2001): Meditationen, übers. v. Achim Russer, Frankfurt am Main: Suhrkamp.

Kristeva, Julia (1990): Fremde sind wir uns selbst, übers. v. Xenia Rajewsky, Frankfurt am Main: Suhrkamp.

Lange, Bernd (2006): Requiem - Drehbuch, Frankfurt am Main: Verlag der Autoren.

Merleau-Ponty, Maurice (1986): Das Sichtbare und das Unsichtbare, übers. v. Regula Guiliani/Bernhard Waldenfels, München: Wilhelm Fink.

Schmid, Hans-Christian (2007): Requiem: Warner Home Video (DVD).

\section{Abbildungsverzeichnis}

Abb. 1: Filmstill aus: Schmid, Hans-Christian (2007): Requiem: Warner Home Video (DVD).

Abb. 2: Raffael (Raffaelo Santi, 1483-1520): Die Verklärung Christi. Vatikanische Museen, Rom, http://epilepsiemuseum.de/deutsch /kunst/transfiguration.html, Stand 04.11.2006

Abb. 3: Filmstill aus: Schmid, Hans-Christian (2007): Requiem: Warner Home Video (DVD). 